and found to have excellent correlation with disease activity index and rSLEDAI scores.

\section{P35 MEASURING DSDNA ANTIBODIES IN THE ELDERLY: POSITIVE RESULTS RARELY INDICATE LUPUS}

Anu Desai, Benjamin Rhodes. Rheumatology Dept., Queen Elizabeth Hospital, Birmingham, UK

\subsection{6/lupus-2020-eurolupus.83}

Background Antibodies to dsDNA are reported to be specific for lupus and are included in the main classification criteria, although pitfalls when used as a 'screening' test in patients with multiple medical symptoms have been described. Lupus is typically diagnosed in the first 50 years of life and clinically active disease is unusual in the elderly. We evaluated dsDNA requests made in elderly patients ( $>80$ years) in our centre.

Methods Review of 23,287 consecutive dsDNA antibody requests made through the University of Birmingham Immunology Lab between 2009 and 2019. Review of available clinical records for patients with positive results.

Results 993 dsDNA antibody requests were made for patients $>80$ years old (mean 84.2 years), representing $0.04 \%$ of total requests. 578 were female, 411 were male and 5 unknown. 138 (13.9\%) requests were made from primary care, $80(8.0 \%)$ from the critical care/emergency department, $623(62.5 \%)$ from medical wards and outpatients and 97 (4.4\%) from rheumatology. The remainder were from neighbouring hospitals. 67 patients (6.7\%) tested positive for dsDNA by ELISA (lab normal range) and 31 (3.1\%) by Crithidia.

Clinical records were available for 58 patients with positive dsDNA antibodies. Three were known to have longstanding SLE. No patients were diagnosed as having SLE at or after the time of dsDNA antibody testing. Sixteen patients had other autoimmune diseases including rheumatoid arthritis, Sjogrens Syndrome, myasthenia gravis, microscopic polyangiitis, polymyalgia rheumatica, bullous pemphigoid and immune thrombocytopenia. Four patients had underlying malignancy (one each of lung, prostate, bladder and colon). The remainder had a mix of infections and general medical presentations.

One hundred randomly selected patients with negative dsDNA antibody were reviewed and no cases of lupus were identified, leading to a calculated specificity of dsDNA antibodies for lupus of only $64.5 \%$.

Conclusions Antibodies to dsDNA are not uncommon in unwell elderly patients presenting to hospital or primary care. They are rarely indicative of SLE. The reported high specificity of dsDNA antibodies for lupus are not applicable in this age group.

\section{P36 USING MACHINE LEARNING TO IDENTIFY AND STRATIFY PATIENTS WITH JUVENILE-ONSET SLE}

George Robinson, Junjie Peng, Anna Radziszewska, Chris Wincup, Hannah Peckham, Meena Naja, David Isenberg, Yiannis Ioannou, Ines Pineda-Torra, Coziana Ciurtin, Elizabeth Jury. Dept. of Medicine, University College London, London, UK

10.1136/lupus-2020-eurolupus.84
Background Juvenile-onset Systemic Lupus Erythematosus (JSLE) is a complex disease characterised by diagnosis and treatment delays. We applied a machine learning (ML) approach to explore new diagnostic signatures for JSLE based on immune-phenotyping data.

Methods Immune-phenotyping of 28 T-cell, B-cell and myeloid-cell subsets in 67 age and sex-matched JSLE patients and 39 healthy controls (HCs) was performed by flow cytometry. A balanced random forest ML predictive model was developed $(10,000$ decision trees). $75 \%$ of sample data was randomly selected as a training set, the remaining $25 \%$ out-of-bag data was used for validation. Reciever operator characteristic, 10fold cross validation, Sparse Partial Least Squares-Discriminant Analysis (sPLS-DA) and linear regression was used to validate the model.

Results In JSLE, a global change in immunological architecture was established compared to HCs: many of the immune cell relationships identified in HCs using correlation comparison analysis were inverted or exacerbated in JSLE, including significantly inverted correlations between intermediate monocytes and memory B-cell populations and CD4/CD8 memory T-cells and B-cell memory in SLE versus HCs. Using immune-phenotyping data a ML model was developed and validated (accuracy $=87.80 \%$ ) showing that JSLE patients could be distinguished from HCs with high confidence using immunological parameters. The top variables contributing to the model included $\mathrm{CD} 19^{+}$unswitched memory B-cells, naïve B-cells, CD14 ${ }^{+}$monocytes and memory T-cell subsets. The 'JSLE immune signature' was also sucessfully validated using sPLS-DA and linear regression. To assess whether the validated signature could be used to further stratify JSLE patients, K-mean clustering was applied. Four JSLE groups each with a distinct immune and clinical profile were identified. Finally, network analysis identified specific clinical features associated with each of the top JSLE immune-signature variables.

Conclusion Using a combined ML approach, a distinct immune signature was identified that discriminated between JSLE patients and HCs and further stratified patients. This signature could have diagnostic and therapeutic implications.

\section{P37 NON-INVASIVE BIOMARKERS OF DISEASE ACTIVITY IN SYSTEMIC LUPUS ERYTHEMATOSUS}

${ }^{1,2}$ Guillermo Ruacho, ${ }^{2}$ Ronaldo Lira-Junior, ${ }^{1}$ Iva Gunnarsson, ${ }^{1}$ Elisabet Svenungsson, ${ }^{2}$ Elisabeth Almer Boström. 'Unit of Rheumatology, Dept. of Medicine, Karolinska University Hospital, Stockholm; ${ }^{2}$ Division of Oral Diseases, Dept. of Dental Medicine. Karolinska Institutet, Stockholm, Sweden

\subsection{6/lupus-2020-eurolupus.85}

Purpose The possibility of exploring other body fluids to identify potential biomarkers of systemic inflammation has emerged as a non-invasive alternative to blood samples for both diagnosis and control of disease activity. We investigated the levels of innate-immunity related biomarkers in saliva, serum, and urine from Systemic Lupus Erythematous (SLE) patients and their correlation to each other and to disease activity.

Methods We included 84 SLE patients and 20 controls from the general population, all participants underwent a thorough clinical examination. Disease activity as measured with the Systemic Lupus Activity Measure (SLAM) and SLE Disease 